\title{
Multiple solutions for a $p$-Laplacian elliptic problem
}

\author{
Jing Zeng ${ }^{1}$ and Shuting $\mathrm{Cai}^{2^{*}}$
}

\author{
"Correspondence: \\ cai_shuting@163.com \\ 2Department of Mathematics and \\ Physics, Fujian Jiangxia University, \\ Fuzhou, 350108, P.R. China \\ Full list of author information is \\ available at the end of the article
}

\begin{abstract}
We consider the following $p$-Laplacian elliptic equation on $W^{1, p}\left(\mathbb{R}^{N}\right)$ :

$-\Delta_{p} u+b(|x|)|u|^{p-2} u=f(|x|, u)$. For certain $f(|x|, u)$, we are interested in the functional on a group invariant subspace, and we obtain the existence of infinitely many radial solutions and non-radial solutions of the equation, which extends the result of (Bartsch and Willem in J. Funct. Anal. 117:447-460, 1993) to the space $W^{1, p}\left(\mathbb{R}^{N}\right)$.
\end{abstract}

Keywords: $p$-Laplacian; infinitely many radial solutions and non-radial solutions; group invariant

\section{Introduction}

The interesting equation

$$
-\Delta_{p} u+b(|x|)|u|^{p-2} u=f(|x|, u), \quad u \in W^{1, p}\left(\mathbb{R}^{N}\right),
$$

originates from different problems in physics and mathematical physics. For $p=2$, problem (1.1) is interpreted as a stationary state of the reaction-diffusion Klein-Gordon equation in chemical dynamics, and Schrödinger equations in finding certain solitary waves.

In the 1980s, people searched for the spherically symmetric solutions of the autonomous equation $\Delta u=g(u)$ (where $g: \mathbb{R} \rightarrow \mathbb{R}$ is continuous and odd in $u$ ). Berestycki-Lions $[1,2]$ advocated it for the first time; they obtained the existence of infinitely many radial solutions of the autonomous equation. Then Struwe [3] got similar results. Gidas et al. [4] further demonstrated that any positive solution of the equation with some properties must be radial.

Then Bartsch-Willem [5] found an unbounded sequence of non-radial solutions of (1.1) in $H^{1}\left(\mathbb{R}^{N}\right)$ with $p=2$, under the assumption that $b$ and $f$ satisfy certain growth conditions and $f$ is odd in $u$.

In recent years, the existence and structure of solutions for the $p$-Laplacian equation has found considerable interest, and different approaches have been developed. Bartsch-Liu [6] studied the $p$-Laplacian problem

$$
-\Delta_{p} u=f(x, u), \quad u \in W_{0}^{1, p}(\Omega)
$$

on a bounded domain $\Omega \subset \mathbb{R}^{N}$ with smooth boundary $\partial \Omega$, provided that the nonlinearity $f$ is superlinear and subcritical. They proved (1.2) has a pair of a subsolution and a supersolution. In [7] they studied problem (1.2) on a bounded domain $\Omega \subset \mathbb{R}^{N}$, with $p>1$ 
arbitrary, and proved a nodal solution provided that $f: \Omega \times \mathbb{R} \rightarrow \mathbb{R}$ is subcritical and superlinear. Infinitely many nodal solutions are obtained if, in addition, $f(x,-t)=-f(x, t)$.

Furthermore Jiu-Su [8] applied Morse theory to study the existence of nontrivial solutions of $p$-Laplacian type Dirichlet boundary value problems. Agarwal-Perera [9] obtained two positive solutions of singular discrete $p$-Laplacian problems using variational methods. Chabrowski-Fu [10] studied the $p$-Laplacian problem:

$$
-a(x) \Delta_{p} u+b(x)|u|^{p(x)-2} u=f(x, u)
$$

on a bounded domain of $\mathbb{R}^{N}$ with Dirichlet boundary condition, where $1<p_{1} \leq p(x) \leq$ $p_{2}<N\left(p_{1}, p_{2}\right.$ are positive constants). They applied the mountain pass theorem to prove the existence of solutions in $W_{0}^{1, p(x)}(\Omega)$ for the equation in the superlinear and sublinear cases.

For (1.1), Drábek-Pohozaev [11] proved the existence of multiple positive solutions of quasilinear problems (1.1) of second order by using the fibering method. They considered solutions both in the bounded domain $\Omega \subset \mathbb{R}^{N}$ and in the whole space $\mathbb{R}^{N}$. Moreover, De Nápoli-Mariani [12] introduced a notion of uniformly convex functional that generalizes the notion of uniformly convex norm. They proved the existence of at least one solution of (1.1), and the existence of infinitely many solutions under further assumptions.

In the present paper, we aim to find the existence of infinitely many radial and non-radial solutions of problem (1.1), and extend the result of [5] to the space $W^{1, p}\left(\mathbb{R}^{N}\right)$.

A direct extension to the case $p \neq 2$ is faced with serious difficulties. First the energy functional associated to (1.1) is defined on $W^{1, p}\left(\mathbb{R}^{N}\right)$, which is not a Hilbert space for $p \neq 2$. Another difficulty is the lack of a powerful regularity theory. For the Laplace operator there exists a sequence of Banach spaces $E_{0} \hookrightarrow E_{1} \hookrightarrow \cdots \hookrightarrow E_{n}$ with $W^{1,2} \hookrightarrow E_{n}$ and $E_{0} \hookrightarrow C^{1}$. But the imbedding $W^{1, p}\left(\mathbb{R}^{N}\right) \hookrightarrow L^{q}\left(\mathbb{R}^{N}\right)\left(p<q<p^{*}:=N p /(N-p)\right.$, for $\left.1<p<N\right)$ is not compact.

We study the functional on a group invariant subspace $\left\{u \in W^{1, p}\left(\mathbb{R}^{N}\right) \mid g u(x)=u\left(g^{-1} x\right)=\right.$ $u(x), g \in O(N)\}$ (where $O(N)$ is the group of orthogonal linear transformations in $\mathbb{R}^{N}$ ), then we apply the principle of symmetric criticality [13, Theorem 5.4] and the fountain theorem to obtain the existence of multiple solutions.

\section{The main results and preliminaries}

This paper is devoted to the study of infinitely many radial and non-radial solutions for a $p$-Laplacian equation:

$$
-\Delta_{p} u+b(|x|)|u|^{p-2} u=f(|x|, u), \quad u \in W^{1, p}\left(\mathbb{R}^{N}\right),
$$

where $\Delta_{p} u=\operatorname{div}\left(|\nabla u|^{p-2} \nabla u\right)(1<p<N), b(r) \in C([0, \infty), \mathbb{R})$ is bounded from below by a positive constant $a_{0}$. The growth condition of $f(|x|, u)$ will be given in the following.

The corresponding functional is

$$
J(u):=\int_{\mathbb{R}^{N}}\left(\frac{1}{p}|\nabla u|^{p}+\frac{1}{p} b(|x|)|u|^{p}-F(|x|, u)\right) d x,
$$

where $F(|x|, u)=\int_{0}^{u} f(|x|, t) d t, u \in W^{1, p}\left(\mathbb{R}^{N}\right)$.

We require the following assumptions on the nonlinearity $f$ : 
(f $\left.\mathrm{f}_{1}\right) f \in C([0,+\infty) \times \mathbb{R}, \mathbb{R})$, and $f(r, t)=o(|t|)$ for $t \rightarrow 0$, uniformly on $[0,+\infty)$.

$\left(\mathrm{f}_{2}\right)$ There exists $\mu>p$ such that for all $r \geq 0, t \in \mathbb{R}, 0<\mu F(r, t) \leq t f(r, t)$.

$\left(\mathrm{f}_{3}\right)$ For $1<p<N$, and $p<q<p^{*}$, there exists a constant $C>0$, such that for any $r \geq 0$, $t \in \mathbb{R},|f(r, t)| \leq C|t|^{q-1}$.

The main result of this paper is as follows.

Theorem 2.1 If $N \geq 2$, the assumptions $\left(\mathrm{f}_{1}\right)-\left(\mathrm{f}_{3}\right)$ hold, and $f$ is odd in $u$, then problem (1.1) possesses infinitely many radial solutions.

Theorem 2.2 Suppose $N=4$ or $N \geq 6$, if the assumptions $\left(\mathrm{f}_{1}\right)-\left(\mathrm{f}_{3}\right)$ hold and $f$ is odd in $u$, then for problem (1.1) there exist infinitely many non-radial solutions.

Remark 2.3 The assumptions $\left(f_{1}\right)-\left(f_{3}\right)$ are from [5]. Bartsch-Willem [5] considered the existence of non-radial solutions for the Euclidean scalar field equation $-\Delta u+V(|x|) u=$ $f(|x|, u)\left(u \in H^{1}\left(\mathbb{R}^{N}\right)\right)$.

$\left(\mathrm{f}_{2}\right)$ means that the nonlinearity $f$ is superlinear, and $\left(\mathrm{f}_{3}\right)$ means that $f$ is subcritical. These two conditions enable us to use a variational approach for the study of (1.1).

Condition $\left(\mathrm{f}_{2}\right)$ corresponds to the standard superlinearity condition of AmbrosettiRabinowitz in the case $p=2$. In the case $p=2$ without the assumption $\left(\mathrm{f}_{3}\right)$, the above theorems may not be true. It can be seen from Pohozaev's identity for $p$-Laplacian equations that (1.1) has only a trivial solution $u=0$.

Remark 2.4 If $N=5$ we fail to define the action of $G_{3}$ in the proof of Theorem 2.2.

We shall use the norm

$$
\|u\|_{p}:=\left(\int_{\mathbb{R}^{N}}\left(|\nabla u|^{p}+b(|x|)|u|^{p}\right) d x\right)^{\frac{1}{p}} .
$$

We denote $E:=W^{1, p}\left(\mathbb{R}^{N}\right)$ is the completion of $D\left(\mathbb{R}^{N}\right)$ with the norm $\|\cdot\|_{p}$, where $D\left(\mathbb{R}^{N}\right):=$ $\left\{u \in C^{\infty}\left(\mathbb{R}^{N}\right) \mid \operatorname{supp}(u)\right.$ is a compact subset in $\left.\mathbb{R}^{N}\right\}$. Denote by $|\cdot|_{p}$ the usual norm in $L^{p}\left(\mathbb{R}^{N}\right)$.

Let $G_{1}=O(N)$ be the group of orthogonal linear transformations in $\mathbb{R}^{N}, G_{2}=\mathbb{Z}_{2}$, and $E_{G_{1}}:=\left\{u \in W^{1, p}\left(\mathbb{R}^{N}\right) \mid g u(x)=u\left(g^{-1} x\right)=u(x), g \in O(N)\right\}$.

Throughout this paper, we will use $C$ and $C_{i}$ to represent various positive constants.

Now, we recall some definitions for the action of a topological group and the fountain theorem.

Definition 2.5 ([14, Definition 1.27]) The action of a topological group $G$ on a normed space $Z$ is a continuous map $G \times Z \rightarrow Z:[g, z] \longmapsto g z$, such that for all $g, h \in G, z \in Z$,

$$
1 \cdot z=z, \quad(g h) z=g(h z), \quad z \longmapsto g z \quad \text { is linear. }
$$

The action is isometric if $\|g z\|=\|z\|$.

The set of invariant points is defined by Fix $G:=\{z \in Z ; g z=z, \forall g \in G\}$. A set $A \subset Z$ is $G$-invariant if $g A=A$ for every $g \in G$. A function $\varphi: Z \rightarrow \mathbb{R}$ is $G$-invariant if $\varphi \circ g=\varphi$ for every $g \in G$.

A map $f: Z \rightarrow Z$ is $G$-equivariant if $f \circ g=g \circ f$, for every $g \in G$. 
Definition 2.6 ([15, p.99]) Suppose $Z$ is a $G$-Banach space, that is, there is a $G$ isometric action on $Z$. Let $\mathcal{A}=\{A \subset Z \mid A$ is closed and $g A=A$, for any $g \in G\}$ be the set of $G$-invariant subsets of $Z$, and $\Gamma=\left\{h \in C^{0}(Z, Z) ; h \circ g=g \circ h\right.$, for all $\left.g \in G\right\}$ be the class of $G$-equivariant mappings of $Z$.

Definition 2.7 ([14]) Let $Z$ be a Banach space, $I \in C^{1}(Z, \mathbb{R})$ and $c \in \mathbb{R}$. The functional $I$ satisfies the $(P S)_{c}$ condition if any sequence $\left\{z_{n}\right\} \subset Z$ such that

$$
I\left(z_{n}\right) \rightarrow c, \quad I^{\prime}\left(z_{n}\right) \rightarrow 0, \quad \text { as } n \rightarrow \infty,
$$

has a convergent subsequence.

Theorem 2.8 (Fountain theorem [14, Theorem 3.6]) The compact group $G$ acts isometrically on the Banach space $X=\overline{\bigoplus_{j \in N} X_{j}}$, the spaces $X_{j}$ are invariant and there exists a finite-dimensional space $V$ such that for every $j \in N, X_{j} \simeq V$. The action of $G$ on $V$ is admissible.

Let $I \in C^{1}(X, \mathbb{R})$ be an G-invariant functional. If for every $k \in \mathbb{N}$, there exists $\rho_{k}>r_{k}>0$ such that

$\left(\mathrm{A}_{1}\right) a_{k}:=\max _{u \in Y_{k},\|u\|=\rho_{k}} I(u) \leq 0$, where $Y_{k}:=\bigoplus_{j=1}^{k} X_{j}$.

$\left(\mathrm{A}_{2}\right) \quad b_{k}:=\inf _{u \in Z_{k},\|u\|=r_{k}} I(u) \rightarrow \infty$, as $k \rightarrow \infty$, where $Z_{k}:=\overline{\bigoplus_{j=k}^{\infty} X_{j}}$.

$\left(\mathrm{A}_{3}\right)$ I satisfies the $(P S)_{c}$ condition, for every $c>0$.

Then I possesses an unbounded sequence of critical values $c_{k} . c_{k}$ can be characterized as

$$
c_{k}=\inf _{\gamma \in \Gamma_{k}} \sup _{u \in B_{k}} I(\gamma(u))
$$

where $\Gamma_{k}=\left\{\gamma \in C\left(B_{k}, X\right) \mid \gamma\right.$ is equivariant and $\left.\left.\gamma\right|_{\partial B_{k}}=i d\right\}, B_{k}:=\left\{u \in Y_{k} \mid\|u\| \leq \rho_{k}\right\}$.

In fact, for each $k \geq 2$, if $b_{k}>a_{k}$, then there exists a critical value $c_{k}>b_{k}$.

\section{Proof of theorems}

Definition 3.1 ([14, Definition A.3]) On the space $L^{p}\left(\mathbb{R}^{N}\right) \cap L^{q}\left(\mathbb{R}^{N}\right)$, we define the norm

$$
|u|_{p \wedge q}=|u|_{p}+|u|_{q} .
$$

On the space $L^{p}\left(\mathbb{R}^{N}\right)+L^{q}\left(\mathbb{R}^{N}\right)$, we define the norm

$$
|u|_{p \vee q}=\inf \left\{|v|_{p}+|\omega|_{q} \mid v \in L^{p}\left(\mathbb{R}^{N}\right), \omega \in L^{q}\left(\mathbb{R}^{N}\right), u=v+\omega\right\} .
$$

Lemma 3.2 ([14, Theorem A.4]) Assume $1 \leq p, q, r, s<\infty, f \in C([0,+\infty) \times \mathbb{R}, \mathbb{R})$ and $f(|x|, u) \leq C\left(|u|^{\frac{p}{r}}+|u|^{\frac{q}{s}}\right)$, then for every $u \in L^{p}\left(\mathbb{R}^{N}\right) \cap L^{q}\left(\mathbb{R}^{N}\right), f(\cdot, u) \in L^{p}\left(\mathbb{R}^{N}\right)+L^{q}\left(\mathbb{R}^{N}\right)$, the operator

$$
T_{1}: L^{p}\left(\mathbb{R}^{N}\right) \cap L^{q}\left(\mathbb{R}^{N}\right) \rightarrow L^{p}\left(\mathbb{R}^{N}\right)+L^{q}\left(\mathbb{R}^{N}\right): u \longmapsto f(|x|, u)
$$


Lemma 3.3 Let $T_{2}: E_{G_{1}} \rightarrow E_{G_{1}}^{*}$ be the mapping given by

$$
\left\langle T_{2} u, v\right\rangle=\int_{\mathbb{R}^{N}}\left(|\nabla u|^{p-2} \nabla u \nabla v+b(|x|)|u|^{p-2} u v\right) d x,
$$

then $T_{2}$ is bounded and continuous.

Proof By the definition of $T_{2}$,

$$
\begin{aligned}
\left\|T_{2} u\right\|_{E_{G_{1}}^{*}} & =\sup _{\|v\|_{p} \leq 1} \int_{\mathbb{R}^{N}}\left(|\nabla u|^{p-2} \nabla u \nabla v+b(|x|)|u|^{p-2} u v\right) d x \\
& \leq \sup _{\|\nu\|_{p} \leq 1}\left(|\nabla u|_{p}^{p-1}|\nabla v|_{p}+b(|x|)|u|_{p}^{p-1}|v|_{p}\right) \\
& \leq|\nabla u|_{p}^{p-1}+b(|x|)|u|_{p}^{p-1} .
\end{aligned}
$$

Therefore $T_{2}$ is bounded. If $u_{n} \rightarrow \tilde{u}$ in $E_{G_{1}}$, by (3.1), then $\left\|T_{2} u_{n}-T_{2} \tilde{u}\right\|_{E_{G_{1}}^{*}} \rightarrow 0$. Hence $T_{2}$ is continuous.

Lemma 3.4 Suppose the nonlinearity $f$ satisfies $\left(\mathrm{f}_{1}\right)-\left(\mathrm{f}_{3}\right)$, then the functional $J \in C^{1}\left(E_{G_{1}}, \mathbb{R}\right)$, and

$$
\left\langle J^{\prime}(u), v\right\rangle=\int_{\mathbb{R}^{N}}|\nabla u|^{p-2} \nabla u \nabla v d x+\int_{\mathbb{R}^{N}} b(|x|)|u|^{p-2} u v d x-\left\langle\phi^{\prime}(u), v\right\rangle,
$$

where $\left\langle\phi^{\prime}(u), v\right\rangle=\int_{\mathbb{R}^{N}} f(|x|, u) v d x$, here $\phi^{\prime}(u)$ is compact. In addition, each critical point of $J$ is a weak solution of problem (1.1).

Proof By Lemma 3.3, we only need to prove $\phi^{\prime}(u)$ is continuous. By Hölder inequality

$$
\begin{aligned}
\left|\left\langle\phi^{\prime}\left(u_{n}\right), v\right\rangle-\left\langle\phi^{\prime}(u), v\right\rangle\right| & \leq \int_{\mathbb{R}^{N}}\left|f\left(|x|, u_{n}\right)-f(|x|, u)\right||v| d x \\
& \leq\left|f\left(|x|, u_{n}\right)-f(|x|, u)\right|_{p^{\prime} \vee q^{\prime}}|v|_{p \wedge q},
\end{aligned}
$$

where $1 / p+1 / p^{\prime}=1,1 / q+1 / q^{\prime}=1$. If $u_{n} \rightarrow u$ in $E_{G_{1}}$, then $u_{n} \rightarrow u$ in $L^{p}\left(\mathbb{R}^{N}\right) \cap L^{q}\left(\mathbb{R}^{N}\right)$. It follows from $\left(f_{3}\right)$ and Lemma 3.2 that

$$
f\left(|x|, u_{n}\right) \rightarrow f(|x|, u) \quad \text { in } L^{p^{\prime}}\left(\mathbb{R}^{N}\right)+L^{q^{\prime}}\left(\mathbb{R}^{N}\right) .
$$

So

$$
\left\|\phi^{\prime}\left(u_{n}\right)-\phi^{\prime}(u)\right\|_{p}=\sup _{\|v\|_{p} \leq 1}\left|\left\langle\phi^{\prime}\left(u_{n}\right)-\phi^{\prime}(u), v\right\rangle\right| \rightarrow 0 .
$$

Assume $u_{n} \rightarrow u$ in $E_{G_{1}}$. Since $E_{G_{1}} \hookrightarrow L^{q}\left(\mathbb{R}^{N}\right)$ is compact, then $u_{n} \rightarrow u$ in $L^{q}\left(\mathbb{R}^{N}\right)$. By Lemma 3.2 and (3.2), $\phi^{\prime}(u)$ is compact.

Lemma 3.5 ([16, Lemma 2.1]) There exist constants $C_{1}$ and $C_{2}$, such that for all $\xi, \eta \in \mathbb{R}^{N}$, $N \geq 1$, we have

$$
\begin{aligned}
& \left(|\xi|^{p-2} \xi-|\eta|^{p-2} \eta\right)(\xi-\eta) \geq C_{1}(|\xi|+|\eta|)^{p-2}|\xi-\eta|^{2}, \quad \text { if } 1<p<2, \\
& \left(|\xi|^{p-2} \xi-|\eta|^{p-2} \eta\right)(\xi-\eta) \geq C_{2}|\xi-\eta|^{p}, \quad \text { if } p \geq 2 .
\end{aligned}
$$


Lemma 3.6 Let $T_{2}$ be defined in Lemma 3.3. If $u_{n} \rightarrow \tilde{u}$ in $E_{G_{1}}$ and $\left\langle T_{2} u_{n}-T_{2} \tilde{u}, u_{n}-\tilde{u}\right\rangle \rightarrow 0$, then $u_{n} \rightarrow \tilde{u}$ in $E_{G_{1}}$.

Proof If $u_{n} \rightarrow \tilde{u}$ in $E_{G_{1}}$, then $\left\{u_{n}\right\}$ is bounded in $E_{G_{1}}$.

If $p \geq 2$, by Lemma 3.5 ,

$$
\begin{aligned}
\left\langle T_{2} u_{n}-T_{2} \tilde{u}, u_{n}-\tilde{u}\right\rangle= & \int_{\mathbb{R}^{N}}\left(\left|\nabla u_{n}\right|^{p-2} \nabla u_{n}-|\nabla \tilde{u}|^{p-2} \nabla \tilde{u}\right)\left(\nabla u_{n}-\nabla \tilde{u}\right) d x \\
& +\int_{\mathbb{R}^{N}}\left(b(|x|)\left|u_{n}\right|^{p-2} u_{n}-b(|x|)|\tilde{u}|^{p-2} \tilde{u}\right)\left(u_{n}-\tilde{u}\right) d x \\
\geq & C_{3}\left(\left\|u_{n}\right\|_{p}^{p}-\|\tilde{u}\|_{p}^{p}\right)
\end{aligned}
$$

so $u_{n} \rightarrow \tilde{u}$ in $E_{G_{1}}$.

If $1<p<2$, by Lemma 3.5 and the Hölder inequality,

$$
\begin{aligned}
\left\|u_{n}-\tilde{u}\right\|_{p}^{p} \leq & \int_{\mathbb{R}^{N}}\left|\nabla u_{n}-\nabla \tilde{u}\right|^{p}\left(\left|\nabla u_{n}\right|+|\nabla \tilde{u}|\right)^{\frac{p(p-2)}{2}}\left(\left|\nabla u_{n}\right|+|\nabla \tilde{u}|\right)^{\frac{p(2-p)}{2}} d x \\
& +\int_{\mathbb{R}^{N}} b(|x|)\left|u_{n}-\tilde{u}\right|^{p}\left(\left|u_{n}\right|+|\tilde{u}|\right)^{\frac{p(p-2)}{2}}\left(\left|u_{n}\right|+|\tilde{u}|\right)^{\frac{p(2-p)}{2}} d x \\
\leq & \left(\int_{\mathbb{R}^{N}}\left|\nabla u_{n}-\nabla \tilde{u}\right|^{2}\left(\left|\nabla u_{n}\right|+|\nabla \tilde{u}|\right)^{p-2} d x\right)^{\frac{p}{2}} \\
& +\left(\int_{\mathbb{R}^{N}}\left(\left|\nabla u_{n}\right|+|\nabla \tilde{u}|\right)^{p} d x\right)^{\frac{2-p}{2}} \\
& +\left(\int_{\mathbb{R}^{N}} b(|x|)\left|u_{n}-\tilde{u}\right|^{2}\left(\left|u_{n}\right|+|\tilde{u}|\right)^{p-2} d x\right)^{\frac{p}{2}} \cdot\left(\int_{\mathbb{R}^{N}}\left(\left|u_{n}\right|+|\tilde{u}|\right)^{p} d x\right)^{\frac{2-p}{2}} \\
\leq & \left(\frac{1}{C_{1}} \int_{\mathbb{R}^{N}}\left(\left|\nabla u_{n}\right|^{p-2} \nabla u_{n}-|\nabla \tilde{u}|^{p-2} \nabla \tilde{u}\right)\left(\nabla u_{n}-\nabla \tilde{u}\right) d x\right)^{\frac{p}{2}} \cdot C_{3} \\
& +\left(\frac{1}{C_{1}} \int_{\mathbb{R}^{N}} b(|x|)\left(\left|u_{n}\right|^{p-2} u_{n}-|\tilde{u}|^{p-2} \tilde{u}\right)\left(u_{n}-\tilde{u}\right) d x\right)^{\frac{p}{2}} \cdot C_{3} \rightarrow 0 .
\end{aligned}
$$

Lemma 3.7 Assume that $f$ satisfies $\left(\mathrm{f}_{1}\right)-\left(\mathrm{f}_{3}\right)$. Let $\left\{u_{n}\right\} \subset E_{G_{1}}$ be a sequence such that

$$
J\left(u_{n}\right) \rightarrow c, \quad J^{\prime}\left(u_{n}\right) \rightarrow 0, \quad \text { as } n \rightarrow \infty,
$$

then $\left\{u_{n}\right\}$ has a subsequence which converges to a critical point of the functional $J$.

Proof First we show that each sequence $\left\{u_{n}\right\} \subset E_{G_{1}}$ satisfying $J\left(u_{n}\right) \rightarrow c, J^{\prime}\left(u_{n}\right) \rightarrow 0$, as $n \rightarrow \infty$, is bounded. By $\left(f_{2}\right)$ and $\left(f_{3}\right)$,

$$
\begin{aligned}
c+1+\left\|u_{n}\right\|_{p} & \geq J\left(u_{n}\right)-\mu^{-1}\left|J^{\prime}\left(u_{n}\right), u_{n}\right\rangle \\
& =\left(\frac{1}{p}-\frac{1}{\mu}\right)\left\|u_{n}\right\|_{p}^{p}+\int_{\mathbb{R}^{N}} \frac{1}{\mu} f\left(|x|, u_{n}\right) u_{n}-F\left(|x|, u_{n}\right) d x \\
& \geq\left(\frac{1}{p}-\frac{1}{\mu}\right)\left\|u_{n}\right\|_{p}^{p},
\end{aligned}
$$

where $\mu>p$ in the assumption $\left(\mathrm{f}_{2}\right)$, so $\left\{u_{n}\right\}$ is bounded in $E_{G_{1}}$. 
Since $E$ is reflexive, $E_{G_{1}}$ is reflexive, then $\left\{u_{n}\right\}$ has a weakly convergent subsequence. Going if necessary to a subsequence, let $u_{n} \rightarrow \bar{u}$. By Lemma 3.4 $\phi^{\prime}\left(u_{n}\right) \rightarrow \phi^{\prime}(\bar{u})$, and by the definition of $T_{2},\left\langle T_{2} u_{n}, v\right\rangle=\left\langle J^{\prime}\left(u_{n}\right), v\right\rangle+\left\langle\phi^{\prime}\left(u_{n}\right), v\right\rangle$, then $T_{2} u_{n}$ converges. So we assume $T_{2} u_{n} \rightarrow u^{*}$. Observe that

$$
\left\langle T_{2} u_{n}-T_{2} \bar{u}, u_{n}-\bar{u}\right\rangle=\left\langle T_{2} u_{n}-u^{*}, u_{n}-\bar{u}\right\rangle+\left\langle u^{*}-T_{2} \bar{u}, u_{n}-\bar{u}\right\rangle \rightarrow 0 .
$$

By Lemma 3.6, $u_{n} \rightarrow \bar{u}$.

Next we want to show that $\bar{u}$ is a critical point of $J$, i.e. $J^{\prime}(\bar{u})=0$. By Lemma 3.3, $T_{2} u_{n} \rightarrow$ $T_{2} \bar{u}$,

$$
J^{\prime}\left(u_{n}\right)=T_{2} u_{n}-\phi^{\prime}\left(u_{n}\right) \rightarrow T_{2} \bar{u}-\phi^{\prime}(\bar{u})=J^{\prime}(\bar{u}) .
$$

By (3.3), $\bar{u}$ is a critical point of $J$.

Now we give the proof of Theorems 2.1 and 2.2 by applying the fountain theorem and the principle of symmetric criticality. First we recall some properties of Banach space.

According to the results in [17], there exists a Schauder basis $\left\{e_{n}^{\prime}\right\}_{n=1}^{\infty}$ for $E$. Let $e_{n}=$ $\int_{O(N)} e_{n}^{\prime}(g(x)) d \mu_{g}$, then $\left\{e_{n}\right\}_{n=1}^{\infty}$ is a Schauder basis for $E_{G_{1}}$. Since $E_{G_{1}}$ is reflexive, there are $\left\{e_{n}^{*}\right\}_{n=1}^{\infty}$, which are characterized by the relations $\left\langle e_{m}^{*}, e_{n}\right\rangle=\delta_{m, n}$, forming a basis for $E_{G_{1}}^{*}$.

We denote

$$
E_{G_{1}}^{(n)}=\operatorname{span}\left\{e_{1}, \ldots, e_{n}\right\}, \quad E_{G_{1}}^{(n) \perp}=\overline{\operatorname{span}\left\{e_{n+1}, \ldots\right\}},
$$

and define a group action of $G_{2}=\left\{1, \tau_{1}\right\} \cong \mathbb{Z}_{2}$.

Lemma 3.8 If $p<q<p^{*}$, then

$$
\delta_{k}:=\sup _{u \in E_{G_{1}}^{(k) \perp},\|u\|_{p}=1}|u|_{q} \rightarrow 0, \quad \text { as } k \rightarrow \infty
$$

Proof It is clear that $0<\delta_{k+1} \leq \delta_{k}$, so we assume for $\delta \geq 0, \delta_{k} \rightarrow \delta$, as $k \rightarrow \infty$. For every $k \geq 0$, there exists $u_{k} \in E_{G_{1}}^{(k) \perp}$ such that $\left\|u_{k}\right\|_{p}=1$ and $\left|u_{k}\right|_{q}>\frac{\delta_{k}}{2}$. By the definition of $E_{G_{1}}^{(k) \perp}$, $u_{k} \rightarrow 0$ in $E_{G_{1}}$. Since the imbedding $E_{G_{1}} \hookrightarrow L^{q}\left(\mathbb{R}^{N}\right)$ is compact, then $u_{k} \rightarrow 0$ in $L^{q}\left(\mathbb{R}^{N}\right)$. Thus we get $\delta=0$.

Proof of Theorem 2.1 Note that $J$ is $G_{1}$-invariant, by the principle of symmetric criticality [13, Theorem 5.4], any critical point of $\left.J\right|_{E_{G_{1}}}$ is a solution of problem (1.1). $J$ is invariant with respect to the action $G_{2}$.

Now we claim that $\left.J\right|_{E_{G_{1}}}$ satisfies the assumptions of the fountain theorem.

By the assumptions $\left(\mathrm{f}_{1}\right)$ and $\left(\mathrm{f}_{3}\right)$, for $u \in E_{G_{1}}$,

$$
\begin{aligned}
J(u) & =\frac{1}{p}\|u\|_{p}^{p}-\int_{\mathbb{R}^{N}} F(|x|, u) d x \\
& \geq \frac{1}{p}\|u\|_{p}^{p}-\frac{C}{2 q} \int_{\mathbb{R}^{N}}|u|^{q} d x \\
& \geq \frac{1}{p}\|u\|_{p}^{p}-\frac{C \delta_{k}^{q}}{2 q a_{0}}\|u\|_{p}^{q},
\end{aligned}
$$


where $a_{0}>0$ is the lower bound of $b(|x|)$. Choose $r_{k}=\left(2 C q \delta_{k}^{q}\right)^{\frac{1}{p-q}}$, by Lemma 3.8, for $u \in$ $E_{G_{1}}^{(k-1) \perp},\|u\|_{p}=r_{k}, J(u) \geq\left(\frac{1}{p}-\frac{1}{2 q a_{0}}\right)\left(2 C q \delta_{k}^{q}\right)^{\frac{p}{p-q}}$, and as $k \rightarrow \infty$,

$$
b_{k}=\inf _{u \in E_{G_{1}}^{(k-1) \perp},\|u\|_{p}=r_{k}} J(u) \rightarrow \infty
$$

This proves $\left(\mathrm{A}_{2}\right)$.

Now we want to show that the condition $\left(\mathrm{A}_{1}\right)$ is satisfied. By integrating, we obtain from $\left(\mathrm{f}_{2}\right)$ and $\left(\mathrm{f}_{3}\right)$ that, there exist two constants $C_{1}, C_{2}>0$, such that for any $x \in \mathbb{R}^{N}, F(|x|, u) \leq$ $C_{1}|u|^{\mu}+C_{2}|u|^{q}$. Hence,

$$
J(u)=\frac{1}{p}\|u\|_{p}^{p}-\int_{\mathbb{R}^{N}} F(|x|, u) d x \geq \frac{1}{p}\|u\|_{p}^{p}-C_{2} \int_{\mathbb{R}^{N}}|u|^{q} d x-C_{1}|u|_{\mu}^{\mu} .
$$

Since $E_{G_{1}}^{(k)}$ is finite dimensional, all norms are equivalent on $E_{G_{1}}^{(k)}$. Therefore, $\mu>p$ and $q>p$ imply that

$$
\sup _{u \in E_{k},\|u\|_{p} \geq R} J(u) \rightarrow-\infty, \quad \text { as } R \rightarrow \infty .
$$

So there exists $\rho_{k}>r_{k}>0$ such that $\left(\mathrm{A}_{1}\right)$ is satisfied.

$(P S)_{c}$ condition is proved above. By Theorem 2.8, we find, for $k \geq 2$, that

$$
c_{k}=\inf _{\gamma \in \Gamma_{k}} \sup _{u \in B_{k}} I(\gamma(u))
$$

are critical values of the functional $J$. So we can get an unbounded sequence of solutions of (1.1), and the solutions are radial.

Proof of Theorem 2.2 In this proof, we will show that it suffices to find the critical points of $J$ restricted to a subspace of invariant functions. The proof is similar to Theorem 1.31 in [14].

Let $2 \leq m \leq N / 2$ be a fixed integer different from $(N-1) / 2$. The action of $G_{3}:=O(m) \times$ $O(m) \times O(N-2 m)$ on $E$ is defined by $g u(x):=u\left(g^{-1} x\right)$. For $\mathbb{R}^{N}$ is compatible with $G_{3}$, the embedding $E_{G_{3}} \hookrightarrow L^{p}\left(\mathbb{R}^{N}\right)\left(2<p<2^{*}\right)$ is compact (or see [18] for details).

Let $\tau_{2}$ be the involution defined on $\mathbb{R}^{N}=\mathbb{R}^{m} \oplus \mathbb{R}^{m} \oplus \mathbb{R}^{N-2 m}$ by

$$
\tau_{2}\left(x_{1}, x_{2}, x_{3}\right):=\left(x_{2}, x_{1}, x_{3}\right)
$$

The action of $H:=\left\{i d, \tau_{2}\right\}$ on $E_{G_{3}}$ is defined by

$$
h u(x)= \begin{cases}u(x), & h=i d \\ -u\left(h^{-1} x\right), & h=\tau_{2} .\end{cases}
$$

It is clear that 0 is the only radial function on the set

$$
E_{G_{3}, H}:=\left\{u \mid u \in E_{G_{3}}, h u=u, \forall h \in H\right\} .
$$

Moreover, the embedding $E_{G_{3}, H} \hookrightarrow L^{p}\left(\mathbb{R}^{N}\right)$ is compact. As in the proof of Theorem 2.1, we obtain a sequence of non-radial solutions $\pm u_{k}$ of (1.1). 


\section{Competing interests}

The authors declare that they have no competing interests.

\section{Authors' contributions}

Both authors contributed equally. Both authors read and approved the final manuscript.

\section{Author details}

${ }^{1}$ School of Mathematics and Computer Sciences, Fujian Normal University, Fuzhou, 350007, P.R. China. ${ }^{2}$ Department of Mathematics and Physics, Fujian Jiangxia University, Fuzhou, 350108, P.R. China.

\section{Acknowledgements}

The first author is supported by the project of 'Min-Hong Kong cooperation postdoctoral training' funded by Fujian Provincial Civil Service Bureau and the Nonlinear Analysis Innovation Team (IRTL1206) funded by Fujian Normal University. The second author is sponsored by the Scientific Research Foundation for the Returned Overseas Chinese Scholars, State Education Ministry.

Received: 17 April 2014 Accepted: 6 May 2014 Published: 20 May 2014

\section{References}

1. Berestycki, H, Lions, PL: Nonlinear scalar field equations. I. Existence of a ground state. Arch. Ration. Mech. Anal. 82, 313-345 (1983)

2. Berestycki, H, Lions, PL: Nonlinear scalar field equations. II. Existence of infinitely many solutions. Arch. Ration. Mech. Anal. 82, 347-375 (1983)

3. Struwe, M: Multiple solutions of differential equations without the Palais-Smale condition. Math. Ann. 261, 399-412 (1992)

4. Gidas, B, Ni, WM, Nirenberg, L: Symmetry of positive solutions of nonlinear elliptic equations in $\mathbf{R}^{n}$. In: Mathematical Analysis and Applications, Part A. Adv. Math. Supp. Stud., vol. 7, pp. 369-402 (1981)

5. Bartsch, T, Willem, M: Infinitely many nonradial solutions of a Euclidean scalar field equation. J. Funct. Anal. 117, 447-460 (1993)

6. Bartsch, T, Liu, Z: On a superlinear elliptic $p$-Laplacian equation. J. Differ. Equ. 198, 149-175 (2004)

7. Bartsch, T, Liu, Z, Weth, T: Nodal solutions of a p-Laplacian equation. Proc. Lond. Math. Soc. 91, 129-152 (2005)

8. Jiu, QS, Su, JB: Existence and multiplicity results for Dirichlet problems with $p$-Laplacian. J. Math. Anal. Appl. 281, 587-601 (2003)

9. Agarwal, RP, Perera, K: Multiple positive solutions of singular discrete $p$-Laplacian problems via variational methods. Adv. Differ. Equ. 2005(2), 93-99 (2005)

10. Chabrowski, J, Fu, Y: Existence of solutions for $p(x)$-Laplacian problems on a bounded domain. J. Math. Anal. Appl. 306, 604-618 (2005)

11. Drábek, P, Pohozaev, SI: Positive solutions for the $p$-Laplacian: application of the fibrering method. Proc. R. Soc. Edinb., Sect. A, Math. 127, 703-726 (1997)

12. De Nápoli, P, Mariani, MC: Mountain pass solutions to equations of $p$-Laplacian type. Nonlinear Anal. 54, $1205-1219$ (2003)

13. Palais, RS: The principle of symmetric criticality. Commun. Math. Phys. 69, 19-30 (1979)

14. Willem, M: Minimax Theorems. Birkhäuser, Boston (1996)

15. Struwe, M: Variational Methods. Springer, Berlin (2000)

16. Damascelli, L: Comparison theorems for some quasilinear degenerate elliptic operators and applications to symmetry and monotonicity results. Ann. Inst. Henri Poincaré, Anal. Non Linéaire 15, 493-516 (1998)

17. Triebel, H: Interpolation Theory, Function Spaces, Differential Operator. North-Holland, Amsterdam (1978)

18. Lions, PL: Symétrie et compactité dans les espaces de Sobolev. J. Funct. Anal. 49, 315-334 (1982)

10.1186/1687-2770-2014-124

Cite this article as: Zeng and Cai: Multiple solutions for a $p$-Laplacian elliptic problem. Boundary Value Problems 2014, 2014:124

\section{Submit your manuscript to a SpringerOpen ${ }^{\circ}$ journal and benefit from:}

- Convenient online submission

- Rigorous peer review

- Immediate publication on acceptance

- Open access: articles freely available online

- High visibility within the field

- Retaining the copyright to your article 